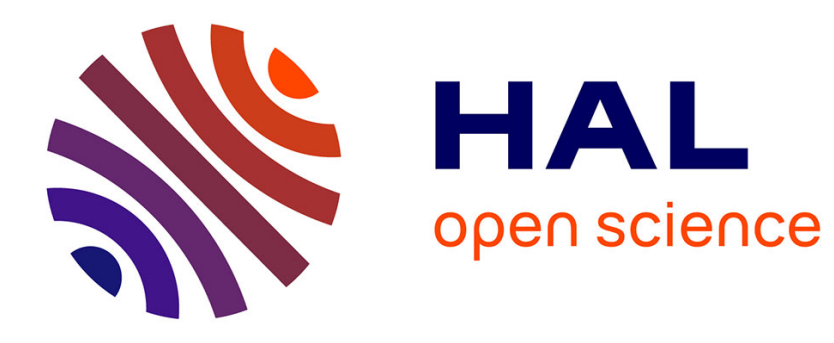

\title{
Complex order van der Pol oscillator
}

\author{
Carla M. A. Pinto, J. A. Tenreiro machado
}

\section{To cite this version:}

Carla M. A. Pinto, J. A. Tenreiro machado. Complex order van der Pol oscillator. Nonlinear Dynamics, 2010, 65 (3), pp.247-254. 10.1007/s11071-010-9886-0 . hal-00640454

\section{HAL Id: hal-00640454 https://hal.science/hal-00640454}

Submitted on 12 Nov 2011

HAL is a multi-disciplinary open access archive for the deposit and dissemination of scientific research documents, whether they are published or not. The documents may come from teaching and research institutions in France or abroad, or from public or private research centers.
L'archive ouverte pluridisciplinaire HAL, est destinée au dépôt et à la diffusion de documents scientifiques de niveau recherche, publiés ou non, émanant des établissements d'enseignement et de recherche français ou étrangers, des laboratoires publics ou privés. 


\title{
Complex order van der Pol oscillator
}

\author{
Carla M.A.Pinto \\ Centro de Matemática da Universidade do Porto and \\ Departament of Matemathics, Institute of \\ Engineering of Porto, Rua Dr. António Bernardino de \\ Almeida, 431, 4200-072 Porto, Portugal \\ cap@isep.ipp.pt
}
J.A. Tenreiro Machado Department of Electrical Engineering, Institute of Engineering of Porto, Rua Dr. António Bernardino de Almeida, 431, 4200-072 Porto, Portugal
jtm@isep.ipp.pt

November 3, 2010

\begin{abstract}
In this paper it is considered a complex order van der Pol oscillator. The complex derivative $D^{\alpha \pm \jmath \beta}$, with $\alpha, \beta \in \mathbf{R}^{+}$is a generalization of the concept of integer derivative, where $\alpha=1, \beta=0$. By applying the concept of complex derivative, we obtain a high-dimensional parameter space. Amplitude and period values of the periodic solutions of the two versions of the complex order van der Pol oscillator, are studied for variation of these parameters. Fourier transforms of the periodic solutions of the two oscillators are also analyzed.

Keywords - van der Pol oscillator, complex order derivative, dynamical behavior

\section{Introduction}

The van der Pol (VDP) oscillator is an ordinary differential equation that has arisen as a model of electrical circuits containing vacuum tubes [45] (re-edited [8]). It produces self-sustaining oscillations in which energy is fed into small oscillations while is removed from large
\end{abstract}


oscillations. This is the first relaxation oscillator appearing in the literature $[44,46]$. It is given by the following second order differential equation

$$
\ddot{x}+\mu\left(x^{2}-1\right) \dot{x}+x=0
$$

Parameter $\mu$ controls the way the voltage flows through the system. For $\mu=0$ this is just a simple linear oscillator. For large values of $\mu$, that is for $\mu \gg 1$, the system exhibits a relaxation oscillation. This means that the oscillator has two distinct phases: a slow recovery phase and a fast release phase (vaccuum tubes quickly release or relax their voltage after slowly building up tension).

This equation has been used in the design of various systems, from biology, with the modeling of the heartbeat [22, 30, 17], the generation of action potentials [21, 20], up to acoustic systems [2] and electrical circuits $[3,12]$. The VDP oscillator has also been used in the context of chaos theory $[9,14,15,16,12]$.

Fractional calculus (FC) has been an important research issue in the last few decades. FC is a generalization of the ordinary integer differentiation and integration to an arbitrary, real or complex, order [34, 43, 28]. Application of FC have been emerging in different and important areas of physics and engineering [35, 27, 26, 31, 39, $41,10,5,42,32]$. Fractional order behavior has been found in areas such as fluid mechanics [29], mechanical systems [13], electrochemistry [33], and biology [11, 1], namely in the modeling of the central pattern generators for animal locomotor rhythms [36, 37].

There are several definitions of fractional derivatives of order $\alpha \in$ $\mathbf{R}$ being three of the most important the Riemann - Liouville, the Grünwald - Letnikov, and the Caputo given by:

$$
\begin{gathered}
D_{t}^{\alpha} f(t)=\frac{1}{\Gamma(n-\alpha)} \frac{d^{n}}{d t^{n}} \int_{a}^{t} \frac{f(\tau)}{(t-\tau)^{\alpha-n+1}} d \tau, \quad n-1<\alpha<n \\
D_{t}^{\alpha} f(t)=\lim _{h \rightarrow 0} \frac{1}{h^{\alpha}} \sum_{h=0}^{\left[\frac{t-a}{h}\right]}(-1)^{k}\left(\begin{array}{c}
\alpha \\
k
\end{array}\right) f(t-k h) \\
D_{t}^{\alpha} f(t)=\frac{1}{\Gamma(\alpha-n)} \int_{a}^{t} \frac{f^{(n)}(\tau)}{(t-\tau)^{\alpha-n+1}} d \tau, \quad n-1<\alpha<n
\end{gathered}
$$

where $\Gamma()$ is the Euler's gamma function, [ ] means the integer part of $x$, and $h$ represents the step time increment. It is also possible to generalize results based on transforms, yielding:

$$
L\left\{D_{t}^{\alpha} f(t)\right\}=s^{\alpha} L\{f(t)\}-\sum_{k=0}^{n-1} s^{k} D_{t}^{\alpha-k-1} f\left(0^{+}\right)
$$


where $s$ and $L$ represent the Laplace variable and operator, respectively.

The definitions demonstrate that fractional derivatives capture the history of the variable, or, by other words, that have memory, contrary to integer derivatives, that are local operators. The Grünwald - Letnikov formulation inspires the numerical calculation of the fractional derivative based on the approximation of the time increment $h$ through the sampling period $T$ and the series truncation at the $r^{\text {th }}$ term. This method is often denoted as Power Series Expansion (PSE) yielding the equation in the $z$ - domain:

$$
Z\left\{D^{\alpha} x(t)\right\} \approx\left[\frac{1}{T^{\alpha}} \sum_{k=0}^{r} \frac{(-1)^{k} \Gamma(\alpha+1)}{k ! \Gamma(\alpha-k+1)} z^{-k}\right] X(z)
$$

where $X(z)=Z\{x(t)\}$ and $z$ and $Z$ represent the $z$-transform variable and operator, respectively. In fact, expression (3) represents the Euler (or first backward difference) approximation in the $s \rightarrow z$ discretization scheme, being the Tustin approximation another possibility. The most often adopted generalization of the generalized derivative operator consists in $\alpha \in \mathbf{R}$. The case of having fractional derivative of complex-order $\alpha \pm \jmath \beta \in \mathbf{C}$ leads to complex output valued results and imposes some restrictions before a practical application. To overcome this problem, it was proposed recently $[18,19,6]$ the association of two complex-order derivatives. In fact, there are several arrangements that produce real valued results. For example, with the real part of two complex conjugate derivatives $D^{\alpha \pm \jmath \beta}$ we get:

$$
\begin{array}{r}
Z\left\{\frac{1}{2}\left[D^{\alpha-j \beta} x(t)+D^{\alpha+j \beta} x(t)\right]\right\} \approx \frac{1}{T^{\alpha}}\left\{\sin \left[\beta \ln \left(\frac{1}{T}\right)\right]\left[\beta z^{-1}+\frac{1}{2} \beta(1-2 \alpha) z^{-2}+\cdots\right]+\right. \\
\left.+\cos \left[\beta \ln \left(\frac{1}{T}\right)\right]\left[-1+\alpha z^{-1}-\frac{1}{2} \beta\left(\alpha^{2}-\alpha-\beta^{2}+\cdots\right)\right]\right\} X(z)
\end{array}
$$

Other combinations and the adoption of a Padé fraction, instead of the series for the approximation, are also possible. Nevertheless, in the sequel it is explored the case of expression (7).

We must remark that it is adopted a scheme of discretized fractional derivative commonly accepted in engineering, but not yet fully investigated from a mathematical view point. Let us recall the basic definitions of Riemann-Liouville (R-L) and Caputo (C) derivatives, in equations (2) and (4), based on integral expressions, and of GrünwaldLetnikov (G-L), in equation (3), based on a series expression suitable for discretization. The $\mathrm{C}$ derivative is a regularization of the $\mathrm{R}-\mathrm{L}$ derivative and their G-L derivative is the series representation of the R$\mathrm{L}$ derivative. In equation (5) the Laplace transform of the R-L deriva- 
tives differs from that of the $\mathrm{C}$ derivative, as shown by Gorenflo and Mainardi [23, 24], subsequently pointed out in the well known treatises on Fractional Calculus by Podlubny [38] and Kilbas-Srivastava and Trujillo [25]. So, in practice it is generalized to complex orders the classical R-L derivative of real order. In case of adopting the Caputo derivative (in view of standard initial conditions), it should be adopted a different Laplace transform (see Gorenflo-Mainardi [23, 24]), and also a modified G-L series representation, because the $\mathrm{C}$ derivative is a regularization of the R- $\mathrm{L}$ derivative in the time origin. For real order less than one the G-L representation of the $\mathrm{C}$ derivative has been adopted in the paper by Gorenflo et al. [24]. As a consequence, the $\mathrm{Z}$ representation in equation (6) in the complex domain should be modified accordingly for the Caputo derivative. While tackling these matters is not straightforward, we must note that the simulations carried in the sequel correspond to steady state responses and that the initial conditions have a minor impact in the resulting charts.

Having these ideas in mind, this paper is organized as follows. In Section 2, we introduce the two approximations of the complex order van der Pol oscillator (CVDP) and we present results from numerical simulations. In Section 3 we outline the main conclusions of this study.

\section{Complex order van der Pol system}

Fractional VDP systems have been studied by many authors $[9,7$, $40,14,4,15,16]$. Their work differ in the approaches considered to express the fractional derivative. Chen et al [9] considered a forced van der Pol equation with fractional damping of the form:

$$
\ddot{x}+\mu\left(x^{2}-1\right) D^{\alpha} x(t)+x(t)=a \sin (\omega t)
$$

where $\mu$ is an endogenous damping parameter, $a$ denotes the amplitude of a periodic forcing, and $\omega$ is the forcing frequency.

Barbosa et al [7] considered the following modified version of the van der Pol equation:

$$
x^{(1+\lambda)}+\alpha\left(x^{2}-1\right) x^{(\lambda)}+x=0
$$

with $0<\lambda<1$.

Tavazoei et al [40] determined the parametric range for which the fractional VDP system studied by Barbosa et al [7] can perform as an undamped oscillator. They also showed that, contrary to the integer order VDP, trajectories in a fractional VDP oscillator do not converge to a unique cycle. 
Ge et al [14] studied the autonomous and non-autonomous fractional van der Pol oscillator. The non-autonomous system in statespace oscillator model is given by:

$$
\begin{aligned}
& \frac{d^{\alpha} x_{1}}{d t^{\alpha}}=x_{2} \\
& \frac{d^{\beta} x_{2}}{d t^{\beta}}=-x_{1}-\mu\left(1-x_{1}^{2}\right)\left(c-a x_{1}^{2}\right) x_{2}+b \sin t
\end{aligned}
$$

where $\alpha$ and $\beta$ are fractional numbers.

To the best knowledge of the authors, little attention has been given to CVDP oscillators. In this paper, we consider the following two complex order state-space models of the VDP oscillator:

$$
\begin{aligned}
& {\left[\begin{array}{c}
\frac{1}{2}\left(D^{\alpha+\jmath \beta}+D^{\alpha-\jmath \beta}\right) x_{1} \\
\dot{x_{2}}
\end{array}\right]=\left[\begin{array}{ll}
0 & 1 \\
-1 & -\mu\left(x_{1}^{2}-1\right)
\end{array}\right]\left[\begin{array}{l}
x_{1} \\
x_{2}
\end{array}\right]} \\
& {\left[\begin{array}{c}
\dot{x_{1}} \\
\frac{1}{2}\left(D^{\alpha+\jmath \beta}+D^{\alpha-\jmath \beta}\right) x_{2}
\end{array}\right]=\left[\begin{array}{ll}
0 & 1 \\
-1 & -\mu\left(x_{1}^{2}-1\right)
\end{array}\right]\left[\begin{array}{l}
x_{1} \\
x_{2}
\end{array}\right]}
\end{aligned}
$$

where $D^{\alpha \pm \jmath \beta}, \alpha, \beta \in \mathbf{R}^{+}$, is a generalization of the concept of the integer derivative, that corresponds to $\alpha=1$ and $\beta=0$.

We adopt the PSE method for the approximation of the complexorder derivative in the discrete time numerical integration. Several experiments demonstrated that it is required a slight adaption to the standard approach based on a simple truncation of the series. In fact, since our objective is to generate limit cycles, the truncation corresponds to a diminishing of the gain [42] and, consequently, leads to difficulties in the promotion of periodic orbits. Therefore, in order to overcome this limitation, we decided to include a gain adjustment factor corresponding to the sum of the missing truncated series coefficients.

The discretisation of the CVDP oscillators (11) and (12) leads to, respectively:

$$
\begin{gathered}
x_{1}(k+1)=\frac{1}{\psi(\beta, \Delta t)}\left(H\left(x_{1}(k)\right)+(\Delta t)^{\alpha} x_{2}(k)\right) \\
x_{2}(k+1)=x_{2}(k)+\Delta t\left(-x_{1}(k)-\mu\left(x_{1}^{2}(k)-1\right) x_{2}(k)\right) \\
x_{1}(k+1)=x_{1}(k)+\Delta t x_{2}(k) \\
x_{2}(k+1)=\frac{1}{\psi(\beta, \Delta t)}\left(H\left(x_{2}(k)\right)+(\Delta t)^{\alpha}\left(-x_{1}(k)-\mu\left(x_{1}^{2}(k)-1\right) x_{2}(k)\right)\right)
\end{gathered}
$$

where $\Delta t=0.0005$ is the time increment, $\psi(\beta, \Delta t)=\cos \left[b \log \left(\frac{1}{\Delta t}\right)\right]$ and function $H\left(x_{i}\right), i=1,2$, results from the Taylor series expansion truncation. 
In Table 1 we depict periodic solutions of systems (13)-(14) for $\alpha=0.4, \beta=0.8$ and $\mu=0.5$. One can observe the appearance of the relaxation oscillation phenomena as $\alpha$ increases (first row of the table). Note that in the case of system (14) this phenomenon is already present for $\alpha=0.4$ and is emphasized for $\alpha=0.8$ (second row of the table).

In Figures 1-2, we show the phase portraits of solutions $\left(x_{1}(t), x_{2}(t)\right)$ of systems (13)-(14) for $\beta=0.8, \mu \in\{0.5,2\}$ and different values of $\alpha$. As expected, the larger the value of $\mu$, the more nonlinear the oscillation becomes. We verify also that we can control the period of the oscillation by varying $\alpha$.

\section{CVDP}

$\alpha=0.4$

$\alpha=0.8$

System (13)
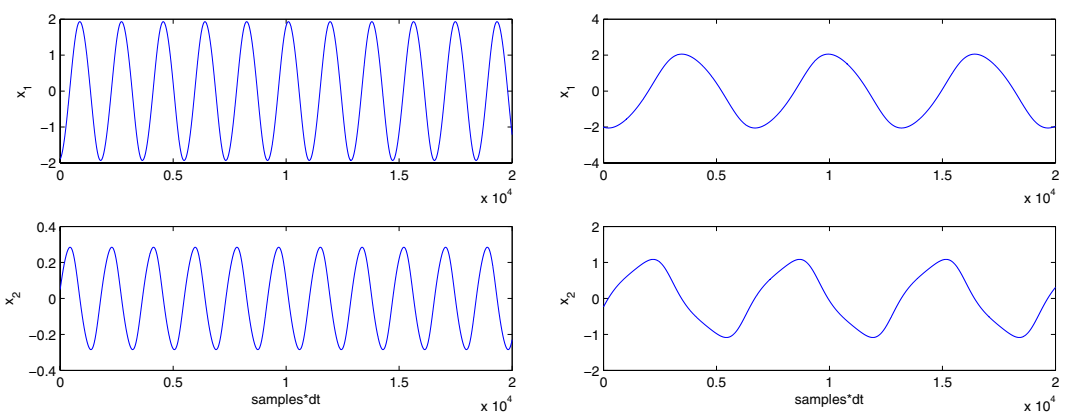

System (14)
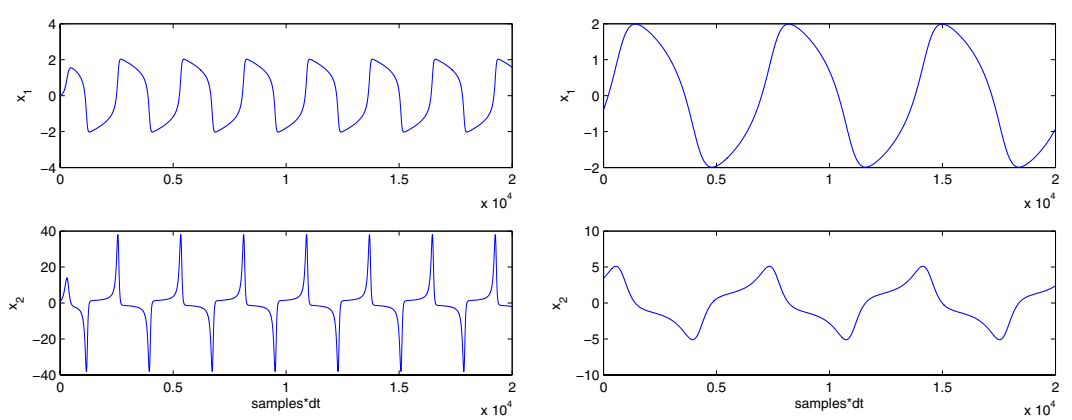

Table 1: Periodic solutions of the CVDP systems (13)-(14) for $\beta=0.8$, $\mu=0.5$ and $\alpha \in\{0.4,0.8\}$.

We now simulate the ordinary differential systems given by expressions (13)-(14) for $\beta=0.8, \alpha \in\{0.2,0.3,0.4,0.5,0.6,0.7,0.8,0.9,1.0\}$, $\mu=\{0.5,1.0,1.5,2\}$, and we measure the amplitude and the period of the solutions. Values of $\alpha \in[0.0,0.2[$ were also considered in the 

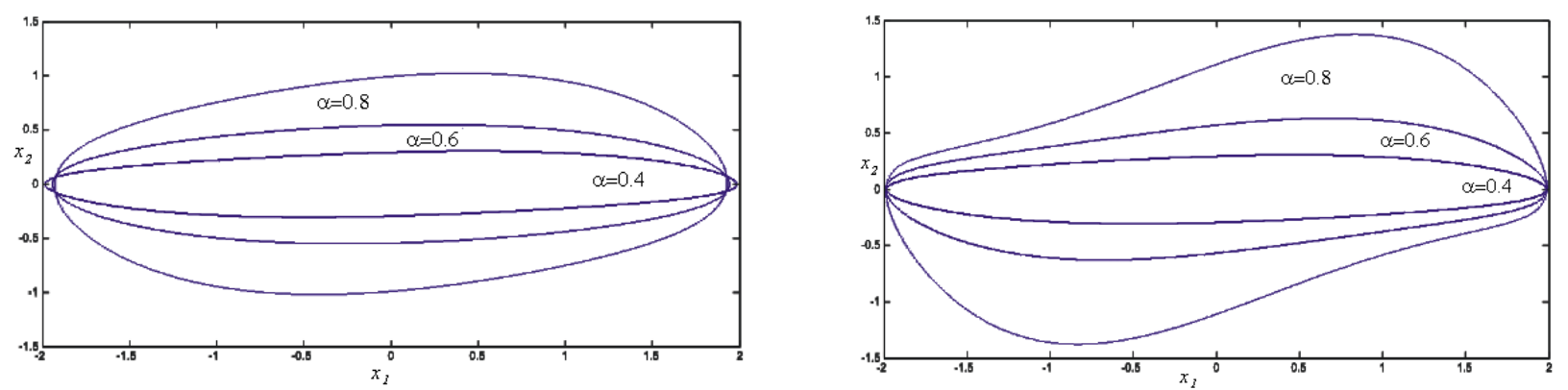

Figure 1: Phase-space solutions $\left(x_{1}(t), x_{2}(t)\right)$ of the CVDP system (13) for $\alpha \in\{0.4,0.6,0.8\}, \beta=0.8$ and $\mu=0.5$ (left) and $\mu=2.0$ (right).
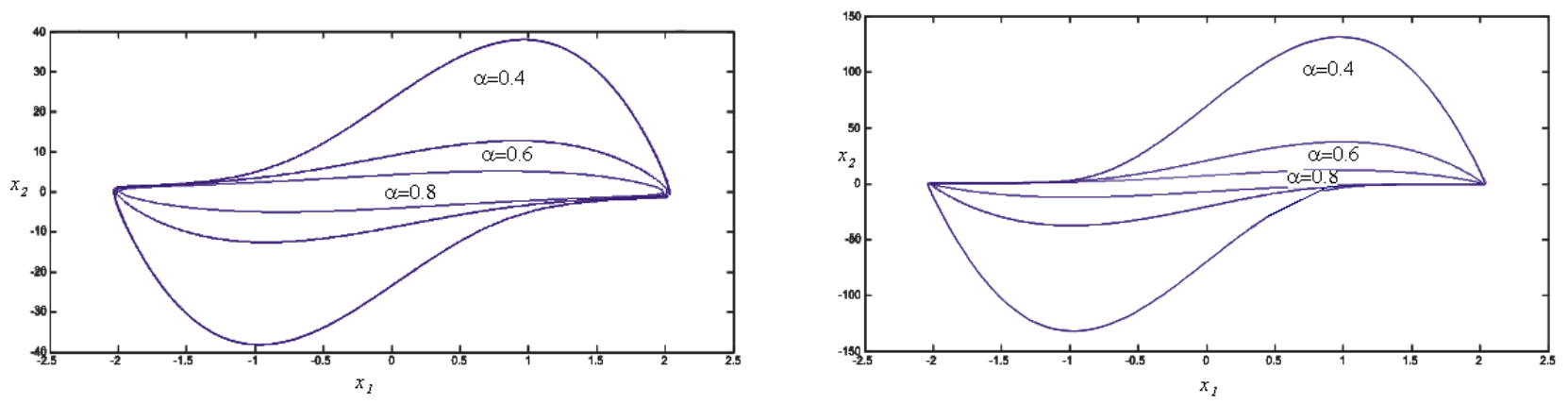

Figure 2: Phase-space solutions $\left(x_{1}(t), x_{2}(t)\right)$ of the CVDP system (14) for $\alpha \in\{0.4,0.6,0.8\}, \beta=0.8$ and $\mu=0.5$ (left) and $\mu=2.0$ (right).

simulations. For system (13) we found stable periodic solutions nevertheless for system (14) simulation results led to unstable solutions, so we have decided to omit these values.

Are adopted the initial conditions $x_{1}(1)=0.0, x_{1}(2)=0.005, x_{1}(3)=$ $0.010, x_{1}(4)=0.015, x_{1}(5)=0.02, x_{2}(1)=1.0, x_{2}(2)=1.005, x_{2}(3)=$ $1.010, x_{2}(4)=1.015, x_{2}(5)=1.02$.

Each simulation is executed until a stable periodic solution is found. The amplitude and the period of the solutions versus $\alpha$ are depicted in Figures 3-4. We find that the period increases as $\alpha$ goes from 0.2 to 1.0, in both systems (13)-(14). On the other hand, the amplitude is almost constant. To be precise, the amplitude shows a very tiny increase in system (13) and a very tiny decrease in system (14), as $\alpha$ increases to one.

We now compute the Fourier transforms $\left|\mathcal{F}\left\{x_{1}(t)\right\}\right|$ of the periodic solutions of systems (13) and (14), for $\beta=0.8$ and $\alpha=\{0.3,0.5,0.7,1.0\}$. Figures 5-6 depict the amplitude of the Fourier transforms vs the fre- 
quency $\omega$. The charts demonstrate that the main part of the
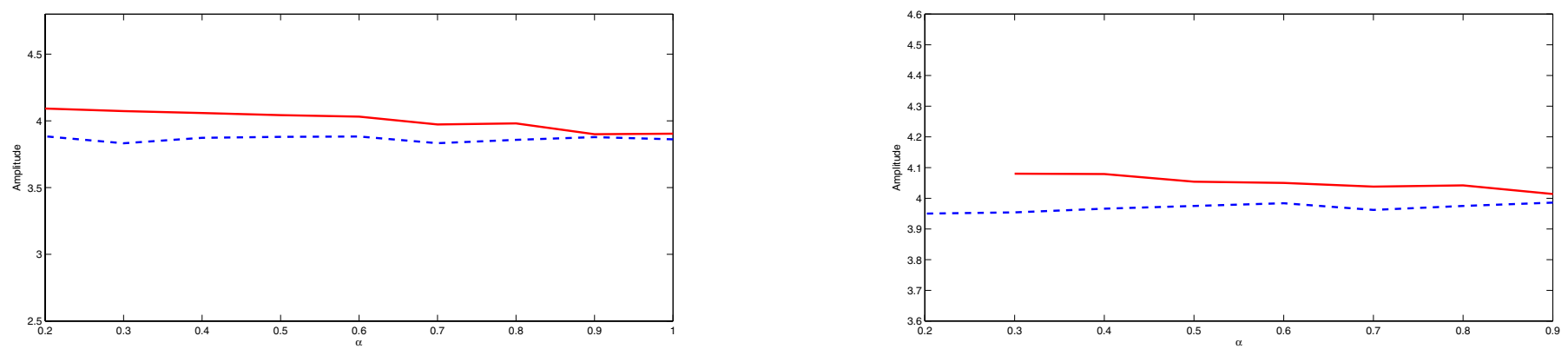

Figure 3: Amplitude of the periodic solutions $x_{1}(t)$ produced by the CVDP oscillators (13) (dashed) and (14) (line) for $\beta=0.8, \alpha \in$ $\{0.2,0.3,0.4,0.5,06,0.7,0.8,0.9,1.0\}$ and $\mu=0.5$ (left) and $\mu=2.0$ (right).
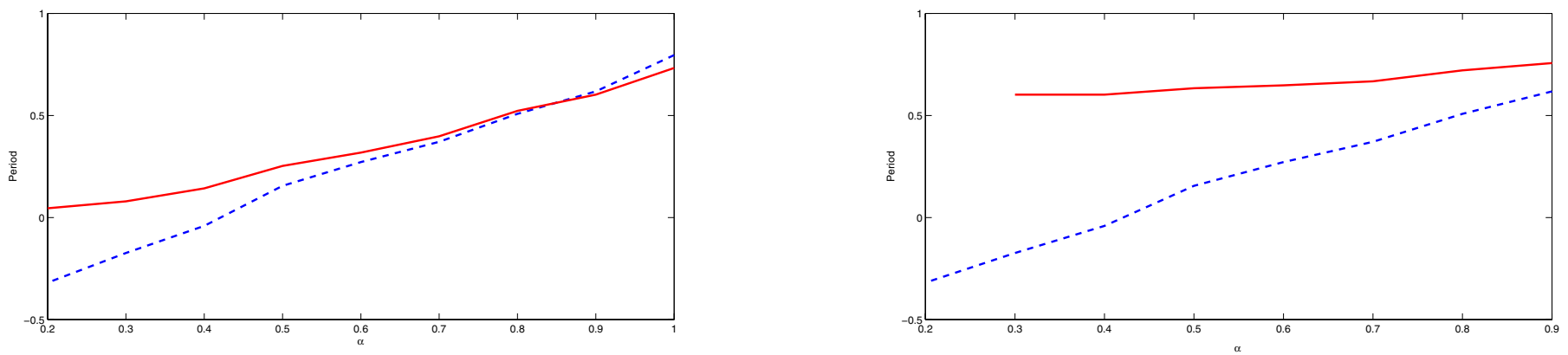

Figure 4: Period of the solutions $x_{1}(t)$ produced by the CVDP oscillators (13) (dashed) and (14) (line) for $\beta=0.8, \alpha \in$ $\{0.2,0.3,0.4,0.5,06,0.7,0.8,0.9,1.0\}$ and $\mu=0.5$ (left) and $\mu=2.0$ (right).

signal energy is concentrated in the fundamental frequency $\omega_{0}$. The remaining energy, located in the higher harmonics increases with $\mu$. Furthermore, it is also observed that the fundamental frequency of the oscillations $\omega_{0}$ varies with $\alpha$ and $\mu$. For the range of tested values, the numerical fitting lead to exponential and rational fraction approximations, for systems (13) and (14), respectively, given by:

$$
\omega_{0} \approx 23.032 \exp (-3.0686 \alpha)
$$

and

$$
\omega_{0} \approx \frac{1}{0.0214+0.29 \mu+(0.7437-0.1202 \mu) \alpha^{2.3158+0.8522 \mu}}
$$



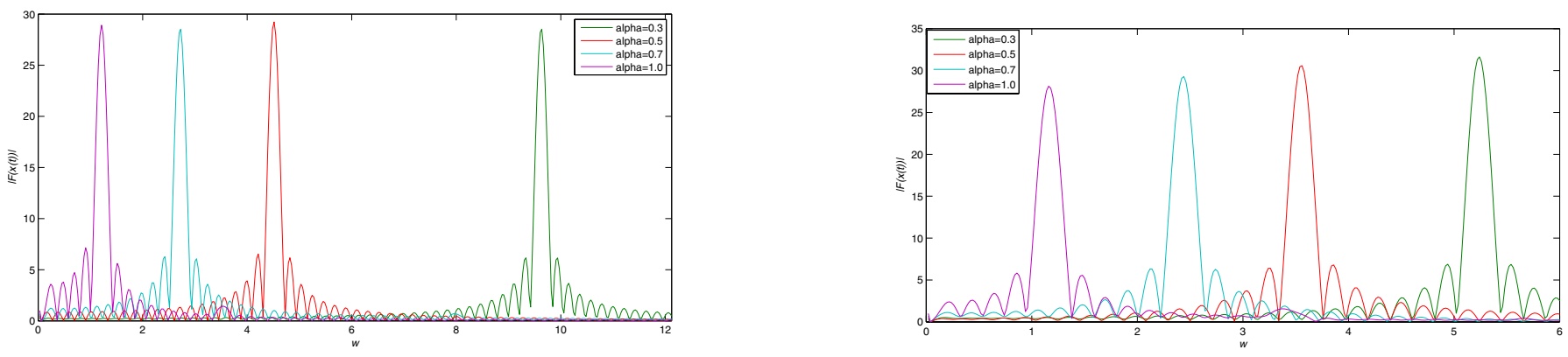

Figure 5: Amplitude of the Fourier transform $\left|\mathcal{F}\left\{x_{1}(t)\right\}\right|$ vs $\omega$ for the CVDP oscilators (13) (left) and (14) (right) with $\beta=0.8, \alpha \in\{0.3,0.5,0.7,1.0\}$ and $\mu=0.5$.
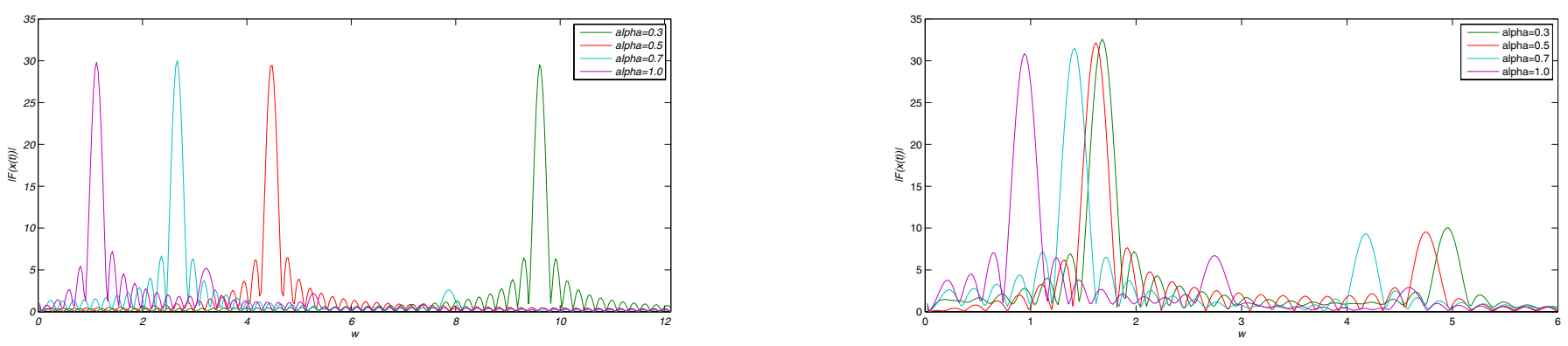

Figure 6: Amplitude of the Fourier transform $\left|\mathcal{F}\left\{x_{1}(t)\right\}\right|$ vs $\omega$ for the CVDP oscilators (13) (left) and (14) (right) with $\beta=0.8, \alpha \in\{0.3,0.5,0.7,1.0\}$ and $\mu=2.0$.

We verify that in the first case the frequency of oscillation is independent of $\mu$, while in the second case it is related both with $\alpha$ and $\mu$.

\section{Conclusions}

In this paper two complex-order approximations to the well-known van der Pol oscillator were proposed. The amplitude and the period of solutions produced by these two approximations were then measured. The imaginary part was fixed while the real component was varied, for two distinct values of parameter $\mu$. It was observed that the waveform period increases as $\alpha$ varies between 0.2 and one. On the other hand, the amplitude values are almost constant as $\alpha$ varies. Moreover, it seems there is a tiny increase in the amplitude of solutions 
for system (13) and a tiny decrease for system (14) as $\alpha$ approaches one.

It was also calculated the Fourier transform $\left|\mathcal{F}\left\{x_{1}(t)\right\}\right|$ for systems (13) and (14). It was verified that the main part of the signal energy is concentrated in the fundamental frequency $\omega_{0}$. The remaining energy, located in the higher harmonics increases with $\mu$. It is also observed that the fundamental frequency of the oscillations $\omega_{0}$ varies with $\alpha$ and $\mu$. For the range of tested values, the numerical fitting lead to exponential and rational fraction approximations for systems (13) and (14), respectively.

\section{Acknowledgments}

CP was supported by Fundação para a Ciência e a Tecnologia (FCT) through the Centro de Matemática da Universidade do Porto www.fc.up.pt/cmup.

\section{References}

[1] Ahmed, E., Elgazzar, A.S. On fractional order differential equations model for nonlocal epidemics Physica A: Statistical Mechanics and its Applications, 379, Issue 2, 607-614, (2007).

[2] Altmann, J. Acoustic and seismic signals of heavy military vehicles for co-operative verification. Journal of Sound and Vibration, 273, Issues 4-5, 713-740, (2004).

[3] Appelbe, B., Rachinskii, D., Zhezherun, A. Hopf bifurcation in a van der Pol type oscillator with magnetic hysteresis. Physica B: Condensed Matter, 403, Issues 2-3, 301-304, (2008).

[4] Attari, M., Haeri, M., Tavazoei, M.S. Analysis of a fractional order Van der Pol-like oscilattor via describing function method. Nonlinear Dynamics. DOI 10.1007/s11071-009-9647-0. (2010).

[5] Baleanu, D. About fractional quantization and fractional variational principles. Communications in Nonlinear Science and $\mathrm{Nu}$ merical Simulations, 14 (6), 2520-2523, (2009).

[6] Barbosa, R.S., Tenreiro Machado, J.A., Silva, M.F. Discretization of Complex-order Algorithms for Control Applications. Journal of Vibration and Control, 14 (9-10), 1349-1361 (2008).

[7] Barbosa, R.S., Tenreiro Machado, J.A. , Vinagre, B.M., Calderón, A.J. Analysis of the Van der Pol Oscillator Containing Derivatives of Fractional Order. Journal of Vibration and Control, 13, Issues 9-10, 1291-1301, (2007). 
[8] Bellman, R., Kalaba, R. Selected Papers on Mathematical Trends in Control Theory, Dover eds: New York. (1964).

[9] Chen, J.-H., Chen, W.-C. Chaotic dynamics of the fractionally damped van der Pol equation. Chaos, Solitons and Fractals, 35 188-198, (2008).

[10] Chen, Y.Q., Moore, K.L. Discretization schemes for fractionalorder differentiators and integrators. IEEE Trans. Circuits and Systems-I: Fundam. Theory Appl. 49 (3), 363-367, (2002).

[11] Darwish, M.A. On quadratic integral equation of fractional orders. Journal of Mathematical Analysis and Applications, 311, Issue 1, 112-119, (2005).

[12] Elabbasy, E.M., El-Dessoky, M.M. Synchronization of van der Pol oscillator and Chen chaotic dynamical system. Chaos, Solitons and Fractals, 36, Issue 5, 1425-1435, (2008).

[13] Ge, Z.-M., Jhuang, W.-R. Chaos, control and synchronization of a fractional order rotational mechanical system with a centrifugal governor. Chaos, Solitons and Fractals, 33, Issue 1, 270-289, (2007).

[14] Ge, Z.-M., Hsu, M.-Y. Chaos in a generalized van der Pol system and in its fractional order system. Chaos, Solitons and Fractals, 33 1711-1745, (2007).

[15] Ge, Z.-M., Hsu, M.-Y. Chaos excited chaos synchronizations of integral and fractional order generalized van der Pol systems. Chaos, Solitons and Fractals, 36 592-604, (2008).

[16] Ge, Z.-M., Zhang, A.-R. Chaos in a modified van der Pol system and in its fractional order systems. Chaos, Solitons and Fractals, 32 1791-1822, (2007).

[17] Glass, L. Theory of Heart, Springer, New York-Heidelberg-Berlin, (1990).

[18] Hartley, T. T., Adams, J. L. and Lorenzo, C. F. Complex-order distributions. Proceedings of the ASME International Design Engineering Technical Conferences and Computers and Information in Engineering Conference, Long Beach, CA, DETC2005-84952, on CD-ROM, (2005).

[19] Hartley, T. T., Lorenzo, C. F., and Adams, J. L. Conjugatedorder differintegrals. Proceedings of the ASME International Design Engineering Technical Conferences and Computers and Information in Engineering Conference, Long Beach, CA, DETC2005-84951, on CD-ROM, (2005). 
[20] FitzHugh R. Impulses and physiological states in theoretical models of nerve membrane. Biophysical J. 1, 445-466, (1961).

[21] FitzHugh R. Mathematical models of threshold phenomena in the nerve membrane. Bull. Math. Biophysics, 17, 257-278, (1955).

[22] FitzHugh R. Motion picture of nerve impulse propagation using computer animation. Journal of Applied Physiology, 25, 628-630, (1968).

[23] Gorenflo, R., and Mainardi, F. Fractional calculus, integral and differential equations of fractional order. In A. Carpenteri and $\mathrm{F}$ Mainardi (Editors), Fractals and Fractional Calculus in Continuum Mechanics, Springer Verlag, (1997).

[24] Gorenflo, R., Mainardi, F., Moretti, D., Pardisi, P. Timefractional diffusion: a discrete random walk approach. Nonlinear Dynamics, 29, No 1-4, 129-143, (2002).

[25] Kilbas, A.A., Srivastava, H.M., Trujillo, J.J. Theory and Applications of Fractional Differential Equations, North Holland Mathematics Studies, 204, Elsevier, (2006).

[26] Machado, J. T. Analysis and design of fractional-order digital control systems. Journal Systems Analysis, Modelling, Simulation, 27, 107-122, (1997).

[27] Mainardi, F. Fractional relaxation-oscillation and fractional diffusion-wave phenomena. Chaos, Solitons and Fractals, 7, 14611477, (1996).

[28] Miller, K. S., Ross, B. An introduction to the fractional calculus and fractional differential equations, John Wiley and Sons, (1993).

[29] Momani, S., Odibat, Z. Analytical approach to linear fractional partial differential equations arising in fluid mechanics. Physics Letters A, 355, Issues 4-5, 271-279, (2006).

[30] Nagumo J., Arimoto S., and Yoshizawa S. An active pulse transmission line simulating nerve axon. Proc IRE. 50 2061-2070, (1962).

[31] Nigmatullin, R. The statistics of the fractional moments: Is there any chance to "read quantitatively" any randomness?. Signal Processing, 86 (10), 2529-2547, (2006).

[32] Nigmatullin, R., Baleanu, D. Is It Possible to Derive Newtonian Equations of Motion with Memory?, Internationa Journal of Theoretical Physics, 49, Issue 4, 701-708, (2010).

[33] Oldham, K. B. Fractional differential equations in electrochemistry. Advances in Engineering Software, 41, Issue 1, 9-12, (2010). 
[34] Oldham, K. B., Spanier, J. The fractional calculus: theory and application of differentiation and integration to arbitrary order. Academic Press (1974).

[35] Oustaloup, A. La commande CRONE: commande robuste d'ordre non entier. Hermes. (1991).

[36] Pinto, C.M.A., Tenreiro Machado, J.A. Complex order biped rhythms. International Journal of Bifurcation and Chaos. Submitted, (2010).

[37] Pinto, C.M.A., Tenreiro Machado, J.A. Fractional Central Pattern Generators for Bipedal Locomotion. Nonlinear Dynamics. Accepted for publication, (2010).

[38] Podlubny, I. Fractional Differential Equations. An Introduction to Fractional Derivatives, Fractional Differential Equations, Some Methods of Their Solution and Some of Their Applications, Academic Press, (1999).

[39] Podlubny, I. Fractional-order systems and PID-controllers. IEEE Transactions on Automatic Control, 44 (1), 208-213, (1999).

[40] M.S. Tavazoei, M. Haeri, M. Attari, S. Bolouki, and M. Siami. More Details on Analysis of Fractional-order Van der Pol Oscillator. Journal of Vibration and Control 15 (6), 803-819 (2009).

[41] Tenreiro Machado, J. Discrete-time fractional-order controllers. Journal of Fractional Calculus and Applied Analysis, 4, 47-66, (2001).

[42] Tenreiro Machado, J. A. Fractional Derivatives: Probability Interpretation and Frequency Response of Rational Approximations. Communications in Nonlinear Science and Numerical Simulations, Elsevier, 14, Issues 9-10, 3492-3497, SeptemberOctober, (2009).

[43] Samko, S. G., Kilbas, A. A., Marichev, O. I. Fractional integrals and derivatives: theory and applications. Gordon and Breach Science Publishers (1993).

[44] B. van der Pol. On relaxation-oscillations, The London, Edinburgh, and Dublin Philosophical Magazine and Journal of Science Ser., 7, 2, 978-992, (1926).

[45] B. van der Pol. Forced oscillations in a circuit with nonlinear resistance (receptance with reactive triode), London, Edinburgh and Dublin Phil. Mag., 3, 65-80, (1927).

[46] B. van der Pol e J. van der Mark. Frequency Demultiplication, Nature, 120, 363-364 (1927). 